\title{
ANALYSIS OF INCREASED CORRELATION IN BLIND AUDIO WATERMARK DETECTION
}

\author{
Krishna Kumar S.* and T. V. Sreenivas \\ Department of Electrical Communication Engineering \\ Indian Institute of Science \\ Bangalore - 560012, INDIA.
}

\begin{abstract}
Audio watermarks are often made signal-dependant to keep them inaudible in the host signals. Blind watermark detectors, which do not have access to the unwatermarked signal, may seem handicapped, because an approximate watermark has to be re-derived from the watermarked signal. Referring to the exact watermark scenario as a semi-blind detector, some reduction in performance is anticipated in blind detection over semi-blind detection. An earlier experimental work revealed that the statistical performance of the blind detector is better than that of the semi-blind detector. It is found that the re-derived watermark is better correlated to the host signal and hence, it leads to better detection performance. It is confirmed that this happens only if the embedded watermark is the same as the examined watermark. This paper focuses on an analytical treatment of the problem. The conclusions from this analysis justify the experimental observation. Clues obtained from this investigation will help in better designs of audio watermarking schemes.
\end{abstract}

\section{INTRODUCTION}

Watermarking schemes using correlation based detection are popular in audio watermarking. In general, the watermark detection schemes can be either informed or blind. An informed detector has access to the original unwatermarked (host) signal. An example of informed detection can be found in [1]. A blind detector does not have access to the original host signal. In audio watermarking, blind detection is necessary due to the large data size and deployment patterns. Also, watermarks for audio signals should be signal-dependant, for achieving perceptual transparency. The watermark is shaped so that it remains inaudible when inserted in the host sigfnal. With both of these requirements of blind detection and a signal dependent (shaped) watermark, it appears that a blind detector is handicapped because it cannot know the exact watermark. The usual solution is

This author is a scientist at Centre for Development of Advanced Computing (C-DAC), Trivandrum, India. to use the watermarked signal as a close approximation to the original signal (because of perceptual transparency) and synthesize an approximate watermark at the detector. Such a blind detection scheme is discussed in [2]. Because of the approximation of the watermark, some reduction in performance is anticipated in blind detection systems. However, not many attempts are listed in the literature which investigate the difference in detection performance between the blind and the semi-blind approaches.

The comparison of a blind detector with an informed detector appears unfair. The availability of the host signal at the detector provides a clear advantage to the informed detector, than the availability of exact watermark. Hence, we introduce the term semi-blind to describe those (sometimes hypothetical) schemes, where sufficient information about the host signal is available at the detector for exactly reproducing the watermark, but the host signal itself is not used for detection. We use the semi-blind detector performance as the reference to compare the performance of a blind detector.

An earlier experimental work [3] revealed that the statistical performance of the blind detector is better than that of the semi-blind detector, when applied to a typical correlationbased watermarking scheme, for audio signals. It is found that the re-derived watermark is better correlated to the host signal and hence leads to better detection performance. This paper is an analytical investigation into the same issue.

\section{WATERMARKING SCHEME}

Pseudo random (PR) sequences are good candidates to be watermark sequences. The ownership information can be mapped onto the key for the PR sequence generator. In audio watermarking, the PR sequences are altered to obtain the actual watermark, which is embedded. The spectral and temporal distribution of the watermark energy is shaped such that it remains inaudible in the host signal. A family of sufficiently uncorrelated PR sequences can be generated by a carefully designed PR sequence generator (the details of which are not in the purview of this paper). A correlator- 
detector (matched filter) can be used for watermark detection.

\subsection{Watermark embedding}

Here we address the problem of watermarking of a host vector $\vec{s}$ with a watermark vector $\vec{w}$, both residing in the vector space $\mathcal{R}^{N}$. The watermarked vector $\vec{x}$ may be expressed as

$$
\vec{x}=\vec{s}+\vec{w}
$$

where is the embedding strength. It is required that be large enough to permit reliable detection as weil as small enough that the modification of the host signal by $\vec{w}$ is imperceptible. This leads to an optimum choice of $\vec{w}$ and for a given $\vec{s}$.

\subsection{Watermark synthesis}

Let $\vec{p}$ be the $N$-length $\mathrm{PR}$ sequence generated by a PR sequence generator. The actual waternark sequence $\vec{w}$ is computed from $\vec{p}$ through a spectral and temporal shaping process. Let us denote this process of auditory modeling and shaping, generically, as

$$
\vec{w}=\boldsymbol{\Psi}(\vec{p}, \vec{s})
$$

We denote this process by the term watermark synthesis.

Let the $N$-length vectors $\vec{s}$ and $\vec{p}$ be split into smaller vectors of length $M$ each. This would be required since the length of the watermark $N$, in many cases, can be too large for a meaningful estimate of the auditory masking properties.

Let $\vec{s}_{i} \equiv s_{i}[n] ; 0 \quad n \quad M \quad 1$ be the $i^{\text {th }}$ segment of the signal vector $\vec{s}$ and $\vec{p}_{i} \equiv p_{i}[n]$ be the corresponding segment of the PR sequence. Let $\vec{S}_{i} \cong S_{i}[k] ; 0 \quad k$ $M \quad 1$ and $\vec{P}_{i} \equiv P_{i}[k]$ be their respective $M$-point DFTs. Let $\ddot{w}_{i} \equiv w_{i}[n]$ be the $i^{t h}$ segment of the watermark vector $\vec{w}$ and $\vec{W}_{i} \equiv W_{i}[k]$ be the $M$-pint DFT of $\vec{w}_{i}$

A simple way of spectral shaping of the PR sequence is to force the watermark magnitude spectrum to follow the magnitude spectrum of the host signal. The phase spectrum of the watermark follows the phase spectrum of the PR sequence. That is

$$
W_{i}[k]=\left|S_{i}[k]\right| e^{j L P_{i}[k]}
$$

It may be observed that, $\left\|\overrightarrow{s_{i}}\right\|=\left\|\overrightarrow{w_{i}}\right\|$ i. e. the energy of the watermark is made equal to the energy of the host signal in each frame, thus achieving a (piece-wise) temporal as well as spectral shaping of the watermark energy. The watermark vector $\vec{w}$ is obtained by concatenating the $M$-length vectors $\vec{w}_{i}$.

\subsection{Watermark detection}

The task of a watermark detector is to verify the presence of a particular watermark sequence $\vec{w}$, due to a specific PR sequence $\vec{p}$, in any given vector $\vec{z}$ in the vector space $\mathcal{R}^{N}$. The correlation coefficient [4] between $\vec{z}$ and $\vec{w}$ is computed as

$$
z w=\frac{\vec{z}^{T} \vec{w}}{\|\vec{z}\| \mid\|\vec{w}\|^{\prime}}
$$

where $\|\vec{z}\|=\left(\sum_{n=0}^{N}|z[n]|^{2}\right)^{1 / 2}$ and $\|\vec{w}\|$ is defined likewise. The correlation coefficient is the test statistic for watermark detection under various conditions of the host signal $\vec{s}$; hence $z w$ can be considered as a randorn variable (RV), and a probability density function ( $p d f) f_{z u}$, can be associated with it.

1. Hypothesis 1: Legitimate watermark is present i.e.,

$$
\vec{z}=\vec{x}=\vec{s}+\vec{w}
$$

Hence $z w=x w$. For a variety of $\vec{s}$, we can model ${ }_{x w}$ as a random variable (RV) and a pdf $f_{x w}$ can be associated with it. Let us call this the legitimate pdf.

2. Hypothesis 2: No watermark or Altemate watemark is present i.e.,

$$
\vec{z}=\vec{y}=\vec{s}+\vec{v}
$$

where $\vec{v}$ is any $N$-length watermark vector (including the zero vector), such that $\vec{v} \neq \vec{w}$; i.e., $\vec{v}=\overrightarrow{0}$ or $\vec{v}=$ $\Psi(\vec{q}, \vec{s})$, where $\vec{q}(\neq \vec{p})$, is a different PR sequence of length $N$. Thus, for this hypothesis, $z w=y w$. Again, $y w$ is an RV with a pdf $f_{\gamma, w}$. Let this be the alternate pdf.

The above formulation is valid for the case of semti-blind detection. In the case of blind detection, $\vec{z}$ is used as an approximation to $\vec{s}$ to derive $\overrightarrow{w^{\prime}}=\Psi(\vec{p}, \vec{z})$. This is different from (2) in that it is performed at the detector, whereas (2) is performed at the embedder. The perceptual fidelity constraint on $\vec{z}$ kceps it sufficiently close to $\vec{s}$ so that $\vec{w}^{\prime}$ can be considered a close approximation to $\vec{w}$. In any case, as before we have a legitimate pdf $f_{x: w^{\prime}}$ and an alternate pdf $f_{y, w^{\prime}}$. Now, for detection, we are given one instance of $z w$ (or $z w^{\prime}$ ), which is compared with an optimum threshold

(or '), and one of the two hypotheses is chosen. The parameters of the legitimate and the alternate pdf's decide the optimum detection threshold and the performance. The thresholds must be chosen to minimize the probability of error, appropriately accounting for false positives as well as false negatives.

(From a watermarking system point of view, the correlations computed over several segments of the test signai might be averaged to get an average correlation coefficient 
${ }^{z} w^{\prime}$, before making the decision. The effect of such an averaging may be visualized as a reduction in the variances of the corresponding distributions, with the means remaining unchanged. Thus it results in lesser probability of error for the same detection threshold. The present analysis focuses only on the original (unaveraged) quantities. The results thus obtained may be easily extended for the averaged quantities.)

\section{ANALYSIS}

In the discussions henceforth, we use the following notations:

- $s$ - unwatermarked, host signal

- $x$ - legitimately watermarked signal

- $y$ - alternately watermarked or unwaternarked signal at the detector

- $z$ - a signal at the detector, either legitimately watermarked or alternately watermarked or unwatermarked and with possible distortions

- $p$ - a legitimate PR sequence

- $q$ - an altemate PR sequence

- $r$ - a PR sequence, either legitimate or alternate

- $w \rightarrow$ a legitimate watermark signal, derived from $p$ and $s$

- $v$ - an alternate watermark signal, derived from $q$ and $s$

- $u$ - an arbitrary watermark signal, derived from $r$ and $s$

- $w^{\prime}$ - a watermark signal, synthesized at the detector from $p$ and $z$

- $f_{z u}-$ pdf of the correlation measure $z w$

- $\mu_{z w}$ - mean of the pdf $f_{z w}$

\subsection{Semi-blind Detection}

An arbitrary signal $\vec{z}$ at the detector can be expressed as $\vec{z}=$ $\vec{s}+\vec{u}+\vec{d}$, where $\vec{s}$ is the host signal and $\vec{u}$ is an arbitrary watermark signal (legitimate, alternate or zero), and $\vec{d}$ is the additive distortion. Let $w$ be the legitimate watermark of interest for the detector. The challenge of the detector is due to the variability of unknown $\vec{s}$ and the distortion $\vec{d}$. This is true for both the blind and the semi-blind detectors. Assuming $\vec{d}=\overrightarrow{0}$ for the present, the correlation detection process leads to the following:

$$
\begin{gathered}
z w=\frac{\vec{z}^{T} \vec{w}}{\|\vec{z}\|\|\vec{w}\|}=\frac{(\vec{s}+\vec{u})^{T} \vec{w}}{\|\vec{z}\|^{\|\vec{w}\|}} \\
\frac{\vec{s}^{T} \vec{w}}{\|\vec{z}\|\|\vec{w}\|}+\frac{\vec{u}^{T} \vec{w}}{\|\vec{z}\|\|\vec{w}\|} \\
\triangle z w+\eta_{z w}
\end{gathered}
$$

The first term is the contribution from the correlation of the examined watermark to the original host signal. The second term is the contribution from the correlation of the examined watermark with the embedded watermark, if any. Similarly, following identical steps for the blind detector, we can write:

$$
z w^{\prime}=z w^{\prime}+\eta_{z w^{r}}
$$

where $\vec{w}^{\prime}$ is the e-synthesized watermark at the detector, instead of $\vec{w}$ of the semi-blind case.

Since we have assumed that there is no distortion in the watermarked signal,

$$
\begin{gathered}
z w={ }_{x w}=\frac{\vec{s}^{T} \vec{w}}{\|\vec{x}\|\|\vec{w}\|}+\frac{\vec{w}^{T} \vec{w}}{\|\vec{x}\|\|\vec{w}\|} \\
=\frac{\vec{s}^{T} \vec{w}}{\|\vec{x}\|\|\vec{w}\|}+\frac{\|\vec{x}\|}{\|\vec{w}\|}
\end{gathered}
$$

or

$$
\begin{gathered}
z w=y_{y w}=\frac{\vec{s}^{T} \vec{w}}{\|\vec{y}\|\|\vec{w}\|}+\frac{\vec{v}^{T} \vec{w}}{\|\vec{y}\|\|\vec{w}\|} \\
\frac{\vec{s}^{T} \vec{w}}{\|\vec{y}\|\|\vec{w}\|}
\end{gathered}
$$

because, for well designed PR sequences, the watermark sequences $\vec{u}$ and $\vec{v}$ will be nearly uncorrelated. Because of the fidelity requirements will be of the order of $2^{4}$ to $2^{5}$. Hence it can be observed that $\|\vec{x}\|=\|\vec{y}\| \quad\|\vec{s}\|$. Further, if we choose $\|\vec{w}\|=\| \vec{s} \mid$ (in our scheme, this condition is met exactly if $N=M$ and approximately if $N \neq M$ ), then

$$
\text { zw } \quad w^{w}+
$$

and the pdf $f_{x w 1}$ would be a mean-shifted replica of the pdf $f_{y_{w}}$, with a shift of .

\subsection{Blind Detection Advantage}

From Eq. (11) it is clear that semi-blind detection has a detection margin of " between the two hypotheses. However, we are interested in analyzing the advantage of blind detector over semi-blind case [3]; i.e., the advantage of $z w^{\prime}$ over $z w$. From Eq.(7), it is clear that $z w$ is made of two components: $z w$ and $\eta_{z w}$. An improvement in the second term i.e., $\eta_{z w^{\prime}} \quad \eta_{z w}$ will have much less contribution because of the scaling by . Therefore we compare $z w$ 
and $z w^{\prime}$. We start the artalysis by investigating the difference in the statistical means of $z w$ and $z w^{\prime}$ given as $\mu_{\xi_{x w}} \equiv \mu_{\xi_{x w^{\prime}}} \quad \mu_{\xi_{x w}}$.

We have

$$
z w=\frac{\vec{s}^{T} \vec{w}}{\|\vec{z}\|\|\vec{w}\|}
$$

and

$$
z w^{\prime}=\frac{\vec{s} T \overrightarrow{w^{\prime}}}{\|\vec{z}\|\left\|\overrightarrow{w^{\prime}}\right\|}
$$

It can be shown that $(1 \quad)\|\vec{w}\| \quad\left\|\overrightarrow{w^{\prime}}\right\|(1+)\|\vec{w}\|$. However, from experimental measurements, it was observed that, on the average $\left\|\overrightarrow{w^{\prime}}\right\| \quad 1.01\|\vec{w}\|$, for $=2^{4}$, thus being appreciably away from either of the bounds. Therefore we can assume that $\left\|\overrightarrow{w^{\prime}}\right\| \quad\|\vec{w}\|$. With this assumption, it turns out that $z w$ and $z w^{\prime}$ differ only through their numerators. Hence it may be stated that $\mu_{\xi_{x w}}$ is proportional to $\mathcal{E}\left(\sum_{n=0}^{N} 1 s[n] w^{\prime}[n] \quad \sum_{n=0}^{N} s[n] w[n]\right)$, where $\mathcal{E}$ is the expectation operator. This quantity is investigated below.

Let $S(j \omega)$ and $W(j \omega)$ be the Discrete-time Fourier transforms (DTFT) of $s[n]$ and $w[n]$ respectively. Then

$$
\mathcal{F}(s[n] w[n])=\frac{1}{2} \int^{+} S(j \Omega) W(j \omega \quad j \Omega) d \Omega
$$

where $\mathcal{F}$ is the DTFT operator. It may be noted that

$$
\begin{gathered}
\frac{1}{N} \sum_{0}^{N}(s[n] w[n]) \\
=\left(\frac{1}{2} \int^{+} S(j \Omega) \dot{W}(j \omega j \quad j \Omega) d \Omega\right)_{\psi=0}
\end{gathered}
$$

since the LHS is the average or DC value of $s[n] w[n]$. Thus we have

$$
\sum_{n=0}^{N} s[n] w[n]=\frac{N}{2} \int^{+} S(j \Omega) W(j \Omega) d \Omega
$$

We have

$$
S(j \omega)=|S(j \omega)| e^{j \theta_{S}(j \omega)}
$$

and from the definition of watermark (Eq.(3)),

$$
W(j \omega)=|S(j \omega)| e^{j \theta_{P}(j \omega)}
$$

where $\theta_{S}(j \omega)$ is the host signal phase spectrum and $\theta_{P}(j \omega)$ is the phase spectrum of the legitimate PR sequence $\vec{p}$. (In the watermark synthesis process, the spectral shaping is carried out on frames of length $M$. Thus Eq.(18) is only an approximation, but a useful one for the analysis.) Thus we have

$$
\sum_{0}^{N} s[n] w[n]=\frac{N}{2} \int^{+}|S(j \Omega)|^{2} \cos \left[{ }_{P}(j \Omega)\right] d \Omega
$$

where $p(j \Omega)=\theta_{S}(j \Omega) \quad \theta_{P}(j \Omega)$. This expression results from the conjugate symmetry of the DTFT's of real sequences $s[n]$ and $w[n]$, and the fact that the LHS has to be a real quantity.

The watermark signal at the embedder $u[n]$ is defined by the properties

$$
\begin{aligned}
& |U(j \omega)|=|S(j \omega)|, \forall \omega \\
& \angle U(j \omega)=\theta_{R}(j \omega), \forall \omega
\end{aligned}
$$

where $\theta_{R}(j \omega)$ is the phase spectrum of $\vec{r}$, which is the PR sequence used by the embedder. Thus

$$
U(j \omega)=|S(j \omega)| e^{j \theta_{R}(j \omega)}
$$

The DTFT of the watermarked signal $z[n])$ is given by

$$
Z(j \omega)=S(j \omega)+U(j \omega)
$$

The approximate watermark $w^{\prime}[n]$ re-synthesized from the $z[n]$ is defined by the properties

$$
\begin{aligned}
& \left|W^{\prime}(j \omega)\right|=|Z(j \omega)|, \forall \omega \\
& \angle W^{\prime}(j \omega)=\theta_{P}(j \omega), \forall \omega
\end{aligned}
$$

Form equation 23, we have

$$
\begin{gathered}
|Z(j \omega)|=|S(j \omega)+U(j \omega)| \\
\left.=|S(j \omega)| 1+{ }^{2}+2 \cos { }_{R}(j \omega)\right)^{\frac{1}{2}}
\end{gathered}
$$

where ${ }_{R}(j \Omega)=\theta_{S}(j \Omega) \quad \theta_{R}(j \Omega)$. For $\ll 1$, this expression can be simplified as

$$
|Z(j \omega)|=|S(j \omega)|\{1+\cos [R(j \omega)]\}
$$

Thus,

$$
W^{\prime}(j \omega)=|S(j \omega)|\{1+\cos [R(j \omega)]\} e^{j \theta_{p}(j \omega)}
$$

Thus we have

$$
\begin{gathered}
\sum_{0}^{N} s[n] w^{\prime}[n]=\frac{N}{2} \int^{+} S(j \Omega) W^{\prime}(j \Omega) d \Omega \\
=\frac{N}{2} \int^{+}|S(j \Omega)|^{2} \cos \left[{ }_{P}(j \Omega)\right] d \Omega \\
+\frac{N}{2} \int^{+}|S(j \Omega)|^{2} \cos \left[{ }_{R}(j \Omega)\right] \cos \left[{ }_{P}(j \Omega)\right] d \Omega(29) \\
\text { Therefore, } \\
\mu_{\xi_{2},} \propto \mathcal{E} \sum_{0}^{N} s\left[n ; w^{\prime}[n] \sum_{0}^{N} s[n] w[n]\right) \\
=C\left(\int^{+}|S(j \Omega)|^{2} \cos \left[{ }_{R}(j \Omega)\right] \cos [P(j \Omega)] d \Omega\right)
\end{gathered}
$$

where $\mathrm{C}$ is an arbitrary positive valued constant. 


\subsection{The legitimate case}

In the case of a legitimately watermarked signal, $\vec{z}=\vec{x}$, $\vec{u}=\vec{w}$ and hence $\theta_{R}(j \Omega)=\theta_{P}(j \Omega)$. Thus from Eq.(30), we have

$$
\mu_{\xi_{x+y}}=C \mathcal{E}\left(\int^{+}|S(j \Omega)|^{2} \cos ^{2}\left[{ }_{P}(j \Omega)\right] d \Omega\right)
$$

Since $C,|S(j \Omega)|^{2}$ and $\cos ^{2} \quad P(j \Omega)$ are all positive valued, it may be stated that

$$
\mu_{\xi_{x w}} \propto
$$

and

$$
\mu_{\xi_{x w}}>0
$$

The results obtained from the experiments is in agreement with Eq.(32) and Eq.(33).

\subsubsection{The alternate case}

In the case of an alternately watermarked signal, $\vec{z}=\vec{y}$, $\vec{u}=\vec{v}$ and hence $\theta_{R}(j \Omega)=\theta_{Q}(j \Omega)$. Thus from Eq.(30), we have

$$
\begin{gathered}
\mu_{\xi_{y w}}= \\
C \mathcal{E}\left(\int^{+}|S(j \Omega)|^{2} \cos [Q(j \Omega)] \cos [P(j \omega)] d \Omega\right)
\end{gathered}
$$

where $\quad Q(j \Omega)=\theta_{S}(j \Omega) \quad \theta_{Q}(j \Omega)$. Since $\vec{p}$ and $\vec{q}$ are uncorrelated, it turns out that

$$
\mathcal{E}(\cos [Q(j \Omega)] \cos [P(j \Omega)]) \quad 0
$$

Hence

$$
\mu_{\xi_{y, t}} \quad 0
$$

The results obtained from the experiments is in agreement with Eq.(35).

It may also be noted that, for an unwatermarked signal at the detector $y w^{\prime}$ trivially reduces to $y w$.

\section{SUMMARY OF EXPERIMENTAL OBSERVATIONS}

It can be observed from Fig. 1 that the pdf $f_{x w^{\prime}}$ is almost a mean shifted replica of the pdf $f_{x w}$. It was also observed that there is an appreciable, consistent and positive-valued shift in the legitimate case, whereas the shift in the altemate case is negligibly small and takes both positive and negative values. It is inferred that a watermark synthesized from a legitimately watermarked signal gets better correlated with the host signal, compared to a watermark synthesized from an unwatermarked signal or an alternately watermarked signal. This excess correlation is proportional to the strength

of the watermark in the watermarked signal. It may be noted that the experimental observations and the analytical results agree with each other.

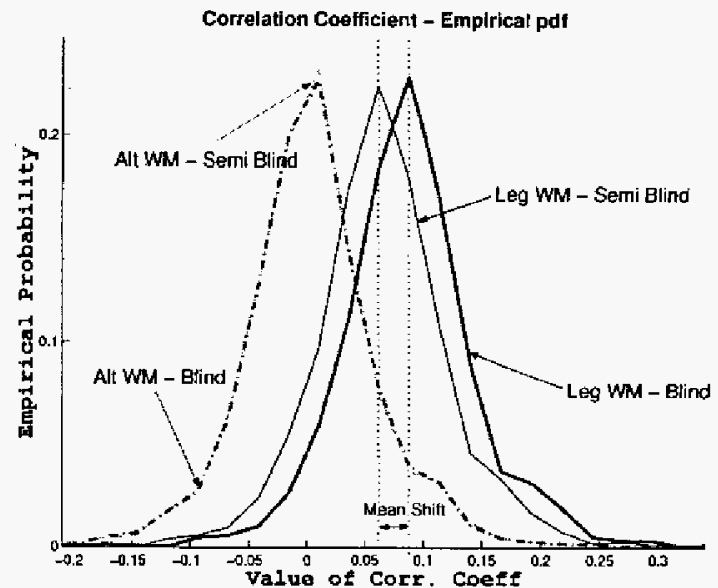

Fig. 1. Empirical pdf's of correlation coefficients: This figure shows the empirical pdf's $f_{x w}$ (Leg WM - Semi Blind), $f_{z w}$ (Leg WM - Blind), $f_{y w}$ (Alt WM - Semi Blind) and $f_{y w}$ (Alt WM - Blind), for $=2^{4}$ and $N=2^{16}$.

\section{CONCLUSIONS}

It was observed that a watermark synthesized from a legitimately watermarked signal gets correlated with the host signal. An analytical explanation for this phenomenon is obtained in this paper. It is observed here that, the correlation of a watermark signal to the host may turn out to be advantageous in some cases. The properties observed from this analysis will be used for better watermark synthesis and hence more effective audio watermarking schemes.

\section{REFERENCES}

[1] M. D. Swanson, B. Zhu, A. H. Tewfik and L. Boney, Robust Audio Watermarking using Perceptual Masking, Elsevier Signal Processing 66(1998), 1998.

[2] P. Bassia, I. Pitas and N. Nikolatdis, Robust Audio Watermarking in the Time Domain, IEEE Trans. Multimedia, June 2001

[3] Krishna Kumar S. and T. V. Sreenivas, Increased Correlation in Blind Audio Watermark Detection - A blessing in Disguise?, submitted to Audio Engineering Society 117 th convention, October 2004.

[4] I. J. Cox, M. L. Miller, and J. A. Bloom, Digital Watermarking, Morgan Kaufmann Publishers, 2002. 